\title{
O papel de adipocinas na relação entre obesidade e resistência à insulina: uma revisão integrativa
}

\section{Role of adipokines in the relationship between obesity and insulin resistance: an integrative review}

\author{
1 Paula Moreira Penna paulapenna_@hotmail.com \\ 1 Helen Hermana Miranda Hermsdorff \\ 2 Margareth Lopes Galvão Saron
}

$\begin{array}{ll}1 & \text { Universidade Federal de Viçosa (UFV) } \\ 2 & \text { Centro Universitário de Volta Redonda - UniFOA }\end{array}$

\section{Resumo}

O presente artigo tem como objetivo revisar artigos que tratem sobre a relação entre a obesidade e a resistência à insulina (RI), com ênfase em adipocinas envolvidas nesse processo. Para tal, foi feita uma revisão de artigos nas bases de dados PubMed, Scielo e Lilacs, em português ou inglês, entre 1993 e 2018, que avaliassem o processo inflamatório na obesidade e seu papel no estabelecimento da RI (desfecho). Nesse contexto, o tecido adiposo é um órgão endócrino e seu aumento resulta na elevação da secreção de citocinas e adipocinas, capazes de modular a RI. Dentre os mecanismos envolvidos, destacam-se inibição do transporte de glicose por intermédio do GLUT-4, diminuição da união da insulina com seus respectivos receptores, inibição da produção de insulina nas células $\beta$ do pâncreas, interferência na sinalização de insulina. Diante do exposto, a identificação de novos biomarcadores e mecanismos envolvidos são primordiais para diagnóstico precoce e sucesso no tratamento das doenças crônicas provenientes do crosstalk entre obesidade e RI.

\section{Palavras-chave}

Adipocinas. Citocinas. Resistina. Leptina. TNF.

\section{Abstract}

This work aims to review articles that deal with the relationship between obesity and insulin resistance (IR), with emphasis on adipokines involved in this process. To this end, articles were reviewed in the PubMed, Scielo and Lilacs databases, in Portuguese or English, between 1993 and 2018, which evaluated the inflammatory process in obesity and its role in establishing IR (outcome). In this context, adipose tissue is an endocrine organ and its increase results in increased secretion of cytokines and adipokines, capable of modulating IR. Among the mechanisms involved are the inhibition of glucose transport through GLUT-4, decreased insulin binding with their respective receptors, inhibition of insulin production in pancreatic $\beta$ cells and interference with insulin signaling. Given the above, the identification of new biomarkers and mechanisms involved are essential for early diagnosis and success in the treatment of chronic crosstalk diseases between obesity and IR.

\section{Keywords}

Adipokines. Cytokines. Resistin. Leptin. TNF.

\section{Como você deve citar?}

PENNA, Paula Moreira; HERMSDORFF, Helen Hermana Miranda; SARON, Margareth Lopes Galvão. O papel de adipocinas na relação entre obesidade e resistência à insulina: uma revisão integrativa. Cadernos UniFOA, Volta Redonda, n. 42, p. 131-141, abril, 2020. 


\section{INTRODUÇÃO}

A prevalência da obesidade vem crescendo de forma acentuada nas últimas décadas, o que fez com que se tornasse um grave problema de saúde pública (COUTINHO, 1999; SCHUSTER, 2010). Os mecanismos fisiopatológicos da obesidade são complexos e diversos, tais como aumento de ácidos graxos livres circulantes, secreção de adipocinas e citocinas, redução da adiponectina, entre outros (HERMSDORFF; MONTEIRO, 2004).

Por sua vez, o acúmulo de gordura corporal, em especial na região abdominal, tem uma forte relação com as alterações metabólicas, incluindo a resistência à insulina (RI). Nesse sentido, a relação de risco entre obesidade, inflamação e RI tem sido muito discutida, visto que tais condições têm sido apontadas como fatores de risco para outras desordens metabólicas (HERMSDORFF; MONTEIRO, 2004; GOBATO et al., 2014; LEE; LAM, 2019).

De fato, um quadro de inflamação subclínica crônica é estabelecido na presença de excesso de gordura corporal (ROCHA E FOLCO, 2011; MORETTO; TORRES, 2016). A diferenciação exacerbada do tecido adiposo resulta em um quadro de microhipoxia, capaz de ativar a sinalização mediada pelo fator de transcrição nuclear (NF-KB) que, por sua vez, é capaz de aumentar a expressão de genes participantes da inflamação e favorecer o recrutamento de macrófagos; todos mecanismos que contribuem para liberação de adipocinas (HERMSDORFF; MONTEIRO, 2004; ROCHA; FOLCO, 2011). Essa liberação de adipocinas, tais como fator de necrose tumoral (TNF), interleucina-6 (IL-6), leptina, resistina, visfatina, apelina têm sido relacionados ao estabelecimento do quadro de RI em indivíduos obesos (ROCHA; FOLCO, 2011; GOBATO et al., 2014; LEE; LAM, 2019).

Diante do exposto, o presente artigo tem como objetivo revisar e discutir a relação existente entre a obesidade e RI, com ênfase em adipocinas e citocinas envolvidas nesse processo.

\section{METODOLOGIA}

A presente revisão integrativa foi conduzida mediante o seguinte questionamento: como as adipocinas e citocinas estabelecem o elo entre a obesidade e RI? As bases de dados utilizadas para a seleção dos artigos foram: PubMed, Scielo e Lilacs. Para tal, os artigos deveriam estar publicados em inglês ou português, no período entre 1993 a 2018.

Inicialmente, as palavras-chave utilizadas para obtenção dos artigos foram: obesidade, resistência à insulina, adipocinas e citocinas, em português ou inglês, conectadas pelos operadores booleanos "AND" ou "OR". Em seguida, cada adipocina ou citocina mencionada foi associada por meio dos mesmos operados booleanos com as palavras-chave: obesidade e resistência à insulina.

Os critérios de inclusão para a seleção dos artigos foram: artigos publicados em inglês ou português, no período entre 1993 a 2018, revisões, estudos clínicos randomizados, estudos observacionais e que tivessem como tema central o processo inflamatório proveniente da obesidade e seu papel no estabelecimento da RI (desfecho), a partir de uma abordagem geral ou específica de alguma citocina ou adipocina.

Foi realizada uma leitura dos títulos e resumos para confirmação da elegibilidade dos artigos para composição da revisão. Em seguida, os artigos considerados compatíveis com o tema abordado foram lidos na íntegra. 
Por fim, todas as informações necessárias para a construção da revisão em questão foram extraídas e posteriormente compiladas, possibilitando o esclarecimento acerca dos mecanismos usados por cada adipocina no estabelecimento do quadro de resistência à insulina.

\section{OBESIDADE, ADIPOCINAS E RI}

\subsection{Leptina}

A clara associação entre obesidade e diabetes mellitus tipo 2 resultou na exploração acerca das interações existentes entre leptina e insulina, devido aos estados de RI e leptina em indivíduos obesos. A leptina parece diminuir a união da insulina com seus respectivos receptores, sendo tal mecanismo verificado a partir de adipócitos de ratos (SEUFERT, 2004). Além disso, esses dois hormônios atuam de forma regulatória, ou seja, a insulina estimula a produção de leptina nos adipócitos e a leptina inibe a produção de insulina nas células $\beta$ do pâncreas (WALDER et al., 1997; SEUFERT, 2004).

A melhora da sensibilidade à insulina e a tolerância à glicose a partir da administração da leptina são atribuídas as seguintes características: capacidade do hormônio em questão de interferir diretamente nos hepatócitos, além da promoção de uma modulação nas vias neuroendócrinas responsáveis por controlar as funções exercidas pela insulina no fígado (LEE et al., 2001).

Ainda se tratando dos efeitos exercidos pela leptina na ação da insulina, sabe-se que a leptina pode potencializar a capacidade da insulina em reduzir a liberação de glicose, por meio da redução da glicólise e, dessa forma, aumentar a fosfoenolpiruvato carboxiquinase (PEPCK), além de diminuir a expressão do gene da glucoquinase (LIU et al., 1998).

Porém, a complexidade que envolve tanto a RI quanto a resistência à leptina, traz consigo uma dificuldade na elucidação dos mecanismos usados por elas, e isso ocorre devido à condição crônica das duas disfunções, que se utilizam de vários órgãos para garantir a homeostase energética e da glicose, além da impossibilidade de estabelecer uma ordem dos eventos ocorridos, por ser inviável avaliar se as disfunções são de caráter primário ou secundário aos eventos iniciais (MORRISON et al., 2009).

A leptina também foi cotada como reguladora da sinalização da insulina, num estudo in vitro com 56 células hepáticas humanas e células de hepatocarcinoma, no qual expressaram o receptor de leptina, e mudanças na sinalização da insulina puderam ser notadas, quando estimuladas com leptina, por meio da diminuição da atividade da tirosina quinase sob receptor de insulina-substrato- 1 do (IRS-1) e diminuição da inibição da insulina na gliconeogênese (COHEN; NOVICK; RUBINSTEIN, 2004).

Ademais, a leptina plasmática se associou diretamente com a insulina plasmática e a RI, medida pelo HOMA-IR em mulheres obesas e não obesas (ALBALA et al., 2000). Moonisha et al. (2017) encontraram valores de HOMA-IR e concentrações de leptina significativamente maiores em indivíduos portadores de diabetes mellitus tipo 2, quando comparados com os valores de referência. Além disso, encontraram uma correlação positiva entre as concentrações de leptina com a insulina plasmática e HOMA.

\subsection{Fator de Necrose Tumoral (TNF)}

Hotalmisligil, Shargill e Spiegelman (1993) constataram que a expressão do TNF no tecido adiposo de roedores obesos é aumentada, quando comparados com controles não obesos. Além disso, identificaram a melhora da tolerância à glicose após neutralizar o TNF nesses roedores. A partir des- 
ses resultados, o TNF tem sido indicado como marcador da relação direta entre a inflamação crônica ocasionada pela obesidade e desenvolvimento da RI.

A presença do TNF no tecido adiposo pode ativar cascatas inflamatórias intracelulares, com serina-quinase como c-Jun $\mathrm{N}$-terminal quinase (JNK) e IKB quinase (IKK), que fosforilam resíduos de serina do IRS-1 e, dessa forma, dificultam a sinalização pela insulina e, consequentemente, inibem sua ação (ROCHA; FOLCO, 2011). Além disso, o TNF atua na regulação do transportador de glicose tipo 4 (GLUT-4) presente nesse tecido, de forma a interferir ainda mais na sensibilidade à insulina. Ademais, essa citocina também inibe a ação da insulina em outros tecidos periféricos (AKASH; REHMAN; LIAQAT, 2017; KIM et al., 2018). Ainda, o TNF pode ocasionar a disfunção das células $\beta$ do pâncreas, por meio da indução de apoptose, em resposta a uma inflamação localizada nas ilhotas pancreáticas, o que irá contribuir de forma considerável para o estabelecimento da RI (REHMAN; AKASH, 2016).

\subsection{Interleucina 6 (IL-6)}

A IL-6 é uma das citocinas mais estudadas, pois suas concentrações são elevadas em indivíduos obesos, tanto no plasma quanto no tecido adiposo branco. É notada uma expressão duas vezes e meia maior em pré-adipócitos do que em adipócitos maduros, o que leva a IL-6 a ter um papel regulador na adipogênese (FAIN et al., 2004; PINTO, 2014).

A RI está relacionada com tal citocina, pois age estimulando a produção de proteínas de fase aguda no fígado, culminando no estado inflamatório crônico ocasionado pela obesidade e a RI surge como uma comorbidade de tal quadro. Além disso, a IL-6 tem uma relação inversamente proporcional com a expressão de IRS-1 e do GLUT-4 nos tecidos adiposo, muscular e hepático (JONES et al., 2001; KERSHAW; FLIER, 2004).

As proteínas que compõem a cascata de sinalização intracelular da insulina são diminuídas, mediante presença de IL-6 e, consequentemente, há redução dos efeitos da insulina. Vale ressaltar também a sua capacidade de diminuir a secreção de adiponectina, uma adipocina que beneficia a ação da insulina e, como já citado anteriormente, tal diminuição ocorre mediante expressão de Supressor de sinalização de citocinas (SOCS-3), que é elevada pela IL-6 (WEYER et al., 2001; LAN et al., 2003).

\subsection{Resistina}

A expressão de resistina tem sido aumentada em indivíduos obesos (SAVAGE et al., 2001; JANKE et al.; AZUMA et al., 2003; DEGAWA-YAMAUCHI et al., 2003; OWECKI et al., 2011; HUANG; ZANG, 2015). Janke et al. (2002) observaram que o transporte de glicose diminuído a partir da insulina e a RI podem ocorrer por intermédio da administração de resistina em roedores obesos. Ademais, constataram uma queda nas concentrações de glicemia e melhora da sensibilidade à insulina mediante administração de anticorpos antirresistina. Em um estudo conduzido por Zaidi e Shirwany (2015), as concentrações séricas de resistina foram mais elevadas em indivíduos diabéticos, quando comparados com o grupo controle. Além disso, houve uma correlação positiva entre a resistina e o IMC em diabéticos e uma relação positiva com IMC e HOMA-IR em indivíduos obesos.

Os mecanismos que levam à associação dessa adipocina com a RI não estão totalmente elucidados. Dentre os mecanismos sugeridos, estão inibição do transporte de glicose por intermédio do GLUT-4, e estímulo para aumento de IL-6, TNF e proteína quimiotática de monócitos-1 (MCP-1) (FU et al., 2006; ZAIDI; SHIRWANY, 2015). 


\subsection{Apelina}

Em humanos e em modelos animais, suas concentrações estão aumentadas na circulação e no tecido adiposo branco na obesidade, sendo sua expressão similar no tecido adiposo subcutâneo e no tecido adiposo intra-abdominal de ratos (XU; TSAO; YUE, 2011). Além disso, o aumento da expressão de apelina foi notado no tecido adiposo (TA) de modelos animais que tinham o desenvolvimento da obesidade somado a RI (BOUCHER et al., 2005).

Estudos em humanos ainda puderam verificar que há aumento das concentrações de apelina em indivíduos obesos, quando apresentavam concomitantemente hiperinsulinemia (CASTAN-LAURELL et al., 2008). Maged et al. (2018) encontraram que crianças obesas possuem concentrações elevadas de apelina, quando comparadas com crianças saudáveis, além de uma correlação significativa entre as concentrações dessa adipocina com concentrações séricas de insulina, HOMA-IR, perfil lipídico e hipertensão. Ademais, outro estudo encontrou que uma dieta hipocalórica diminuiu as concentrações de insulina, apelina e TNF de mulheres obesas, acompanhados de uma diminuição dos receptores de apelina (APJ) no TA (BOUCHER et al., 2005; CASTAN-LAURELL et al., 2008).

Uma das hipóteses que poderia explicar as altas concentrações de apelina, em ratos e humanos obesos é que o aumento seria uma medida compensatória adotada frente ao quadro de RI. Ademais, alguns autores apontam para a possibilidade de um mecanismo regulatório exercido pela insulina sobre a apelina (MORENO-ALIAGA et al., 2008; ALEXIADOU et al., 2012).

Por outro lado, a apelina poderia modular a sensibilidade à insulina, mediante estimulação da fosfoinositídeo 3-quinase (PI3K), que é essencial para o transporte de glicose e, de forma indireta, pode inibir a expressão de NF-KB, promovendo inibição de TNF e, consequentemente, a RI mediada pela citocina (XU; TSAO; YUE, 2011). Contudo, os efeitos da apelina na RI não estão totalmente esclarecidos, de forma que não é possível afırmar se sua superprodução na obesidade otimiza o desenvolvimento da RI ou poderia ser fator protetor para o desenvolvimento de DM2 (MORENO-ALIAGA et al., 2008).

\subsection{Visfatina}

A visfatina é uma adipocina que foi identificada recentemente, portanto sua relação com a obesidade e suas comorbidades precisam ser estudadas de forma mais aprofundada, para haver melhor esclarecimento dos mecanismos que as envolvem nesse contexto (GODOY-MATOS et al., 2014).

Há um paradoxo no que diz respeito aos efeitos fisiológicos da visfatina, pois ao mesmo tempo em que ela facilita a adipogênese, também otimiza os efeitos da insulina, exercendo funções autócrina e parácrina sobre o tecido adiposo visceral. De modo autócrino, facilita a diferenciação e o depósito de gordura e endócrino por modular a sensibilidade à insulina em órgãos periféricos e, nesse sentido, é benéfica para o diabetes, porém ao favorecer o depósito de gordura visceral, contribui para o incremento da obesidade (ZULET et al., 2007).

Seu potencial em reduzir a RI e sua ação insulino-mimética já são conhecidos (MARTOS-MORENO; KOPCHICK; ARGENTE, 2013). Nesse contexto, a visfatina é tida como homóloga a nicotinamida fosforibosiltransferase (NAMPT), enzima responsável por desencadear a síntese de nicotinamida adenina dinucleotídeo (NAD), que atua no funcionamento das células $\beta$ do pâncreas na secreção da insulina. A partir disso, é possível obter uma explicação acerca da associação negativa existente entre visfatina e RI (LUIS et al., 2011). 
É importante esclarecer que não há competição entre a visfatina e a insulina nos receptores de insulina, pois se ligam em sítios diferentes. Ademais, suas concentrações não são alteradas durante os períodos de jejum ou pós-prandiais, como ocorre com a insulina, mantendo-se em concentrações inferiores, se comparado aos da insulina plasmática. Assim seu efeito sobre a glicemia é menor que aquele ocasionado pela insulina. No entanto, não foi possível estabelecer uma melhora da RI mediante tratamento com visfatina, mas ainda assim sua descoberta possibilita uma compreensão mais ampla acerca da obesidade (LUIS et al., 2011).

Ademais, sua presença otimiza as vias inflamatórias, aumentando a síntese da IL-6, TNF e IL$1 \mathrm{~b}$ e estudos indicam sua influência em processos ateroscleróticos e na secreção da insulina (TILG; MOSCHEN, 2008). Uma hipótese poderia ser uma ação indireta da visfatina na RI mediada pelo processo inflamatório (LEITE; ROCHA; BRANDÃO-NETO, 2009).

\subsection{Adiponectina}

Os diversos nomes dados a essa adipocina, como adiponectina (adipoQ), apM1 (do inglês, adipose most abundant gene transcript 1), Acrp30 (do inglês, adipocyte complement-related protein of 30 $\mathrm{kDa}$ ) e GBP28 (do inglês gelatin binding protein of $28 \mathrm{kDa}$ ), se deve ao fato de ter sido descoberta por quatro grupos de estudo que trabalhavam de forma isolada. Sua produção se dá predominantemente pelos adipócitos, sendo a adipocina mais abundante produzida por eles. Acreditava-se que tal função era exclusiva dessas células, porém alguns estudos sugerem que tecidos, como os cardiomiócitos e o músculo esquelético também podem produzi-la (FRAYN et al., 2003; PIÑERO et al., 2005; DAMIANI; DAMIANI, 2006; PETTO et al., 2015).

A adiponectina dispõe de um papel antagônico às demais adipocinas, pois apresenta uma ação anti-inflamatória e antiaterogênica e, dessa forma, torna-se um fator de grande relevância para prevenção de doenças cardiovasculares (DVC), além de ter um valor no prognóstico. Mostra-se capaz de aumentar a sensibilidade à insulina e desempenha um efeito favorável no período pós-prandial, no que diz respeito ao metabolismo de glicose e lipídios (FRAYN et al., 2003; RIBEIRO FILHO et al., 2006; COSTA et al., 2011).

Tal adipocina tem potencial de ativar a expressão de moléculas que aumentam a oxidação de ácidos graxos no músculo esquelético, e reduzem a RI nesse tecido. Além disso, a adiponectina também pode ativar a enzima adenina monofosfato quinase hepática, que resulta na diminuição da produção de glicose hepática (BARBALHO; MCLELLAN; LERARIO, 2007; GUIMARÃES et al., 2007).

Existem evidências que apontam para uma possível aplicação terapêutica do uso da adiponectina, principalmente em casos de RI e diabetes tipo 2, de modo que doses de adiponectina e leptina poderiam ser capazes de reverter a RI de ratos lipoatróficos. Porém, quando usadas separadamente, a reversão do quadro foi parcial (CARVALHEIRA; ZECCHIN; SAAD, 2002; SILVEIRA et al., 2009). 
Figura 1 - Mecanismos envolvidos no estabelecimento da RI em obesos

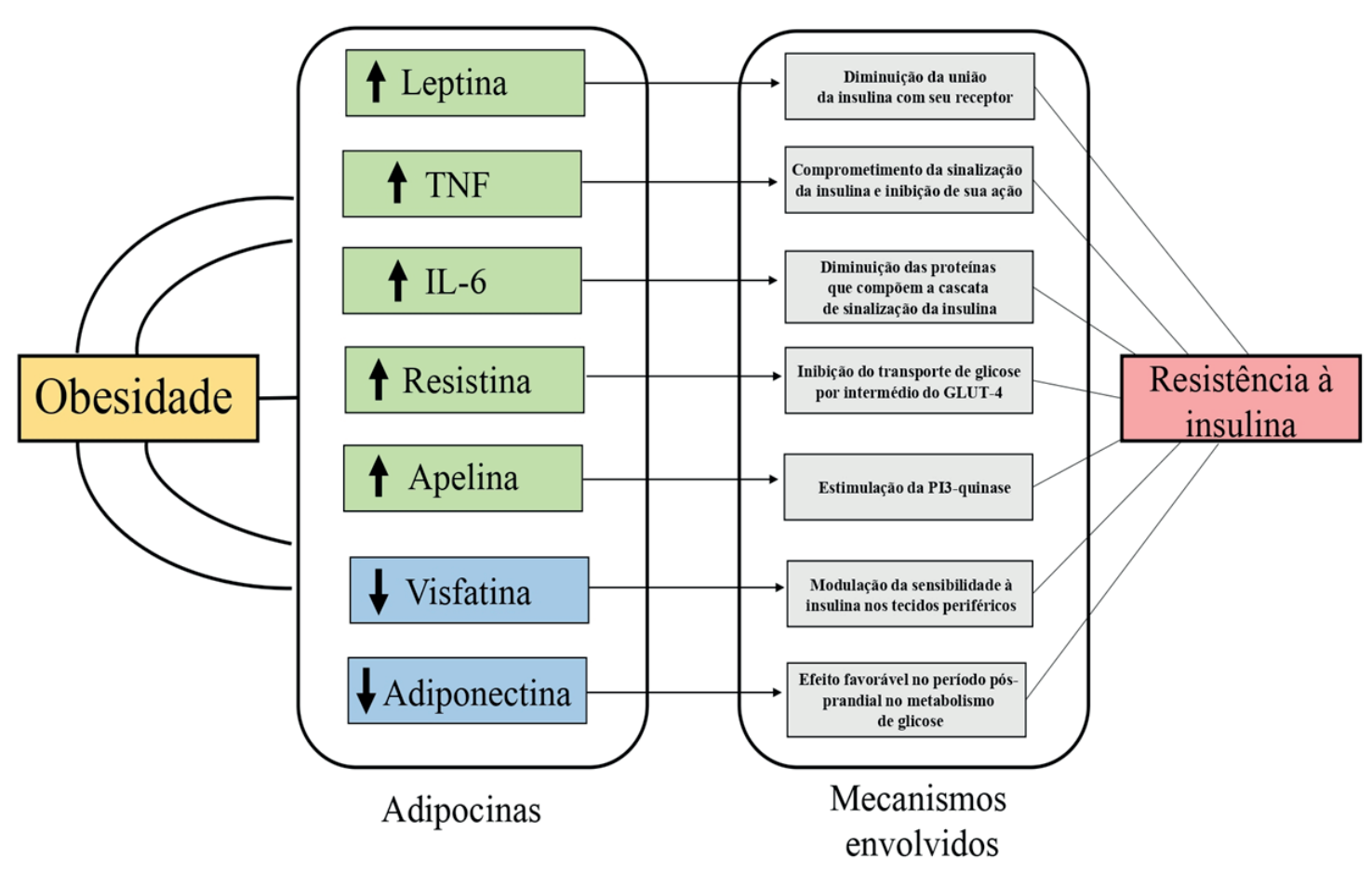

Fonte: dos autores, 2019.

\section{CONSIDERAÇÕES FINAIS}

De modo geral, os resultados apresentados nessa revisão indicam que a liberação de adipocinas, resultantes da diferenciação exacerbada do tecido adiposo na obesidade, atua de forma direta no estabelecimento da RI. Cada uma exerce uma função diferente e envolve mecanismos distintos em todo esse processo (Figura 1). Dentre os mecanismos envolvidos, estão aqueles regulatórios da secreção da insulina e, principalmente os relacionados a sua função sinalizadora de captação de glicose. Diante disso, a identificação de novos biomarcadores (preditores precoces do desenvolvimento da RI) e avaliação dos mecanismos envolvidos (possíveis vias terapêuticas) são primordiais para prevenção e tratamento das doenças crônicas provenientes do crosstalk entre obesidade e RI. 


\section{REFERÊNCIAS}

AKASH, M.S.H.; REHMAN, K.; LIAQAT, A.; Tumor necrosis factor-alpha: Role in development of insulin resistance and pathogenesis of type 2 diabetes mellitus. Journal of Cellular Biochemistry. 2017 v. 119 , sn, p. 1-16, 2017.

ALBALA, C et al. Relación entre leptina e insulina sanguíneas em mujeres chilenas obesas y no obesas. Rev. Méd. v. 128, n. 2, sp, 2000.

ALEXIADOU, K. et al. Differences in plasma apelin and visfatin levels between patients with type 1 diabetes mellitus and healthy subjects and response after acute hyperglycemia and insulin administration. HORMONES. 11(4):444-450, 2012.

AZUMA, K. et al. Correlation between Serum Resistin Level and Adiposity in Obese Individuals. OBESITY RESEARCH. v. 11, n. 8, p. 997-1001, 2003.

BARBALHO, S.M.; MCLELLAN, K.C.P.; LERARIO, A.C. A síndrome metabólica e sua relação com a resistência à insulina, disfunção endotelial e aterogênese. Nutrire. v. 32, n. 1, p. 89-100, 2007.

BOUCHER et al. Apelin, a Newly Identified Adipokine Up-Regulated by Insulin and Obesity. Endocrinology. v. 146, n. 4, p. 1764-1771, 2005.

CARVALHEIRA J.B.C.; ZECCHIN H.G.; SAAD M.J.A. Bases moleculares e fisiologicas da resistencia a insulina. Hipertensão 2002. In: SILVEIRA, M.R. et al. Correlação entre obesidade, adipocinas e sistema imunológico. Revista Brasileira de Cineantropometria e Desempenho Humano. v. 11, n. 4, p. 466-472, 2009.

CASTAN-LAURELL, I. et al. Effect of hypocaloric diet-induced weight loss in obese women on plasma apelin and adipose tissue expression of apelin and APJ. European Journal of Endocrinology v. 158, sn, p. 905-910, 2008.

COHEN, B.; NOVICK, D.; RUBINSTEIN, M.; Modulation of Insulin Activities by Leptin. 1997 in ZAMORAVALDÉS, D.; CHÁVEZ-TAPIA, N.C.; MÉNDEZ-SÁNCHEZ, N. Mecanismos moleculares de resistencia a la insulina. Médica Sur Sociedad de Médicos. v. 11, n. 3, p. 149-159, 2004.

COSTA, M.C. et al. Adiponectina e baixo risco cardiometabólico em obesas. Arquivos Brasileiros de Endocrinologia \& Metabologia. v. 55, n. 2, p. 146-154, 2011.

COUTINHO, W. et al. Consenso Latino Americano sobre Obesidade. São Paulo, 1999. Disponível em: <http://www.abeso.org.br/uploads/downloads/1/5521abe181cb8pdf>. Acesso em 9 mar. 2019.

DAMIANI, D.; DAMIANI, D. Tecido adiposo: depósito de gordura ou um complexo órgão endócrino? Pediatria Moderna. v. 42, n. 2, p. 56-66, 2006.

DEGAWA-YAMAUCHI, M. et al. Serum Resistin (FIZZ3) Protein Is Increased in Obese Humans. The Journal of Clinical Endocrinology \& Metabolism. v. 88, n. 11, p. 5452-5455, 2003.

FAIN, J.N. et al. Comparison of the Release of Adipokines by Adipose Tissue, Adipose Tissue Matrix, and Adipocytes from Visceral and Subcutaneous Abdominal Adipose Tissues of Obese Humans. Endocrinology. v. 145, n. 5, p. 2273-2282, 2004. 
FRAYN, K.N. et al. Integrative physiology of human adipose tissue. International Journal of Obesity. v. 27, sn, p. 875-888, 2003.

FU, Y. et al. Proinflammatory cytokine production and insulin sensitivity regulated by overexpression of resistin in 3T3-L1 adipocytes. Nutrition \& Metabolism. sv, sn, p. 1-10, 2006.

GOBATO, A.O. et al. Síndrome metabólica e resistência à insulina em adolescentes obesos. Ver Paul Pediatr. V. 32, n. 1, p. 55-62, 2014.

GODOY-MATOS, A.F. et al. Adipocinas: uma visão geral dos seus efeitos metabólicos. Revista HUPE. v. 13, n. 1, p. 54-60, 2014.

GUIMARÃES, D.E.D. et al. Adipocitocinas: uma nova visão do tecido adiposo. Revista de Nutrição. v. 20, n. 5, p. 549-559, 2007.

HERMSDORFF, H.H.M.; Monteiro, J.B.R. Gordura Visceral, Subcutânea ou Intramuscular: Onde está o Problema? Arquivos Brasileiros Endocrinologia \& Metabologia. V. 48, n. 6, p. 803-811, 2004.

HOTAMISLIGIL, G.S.; Shargill, N.S.; Spiegelman, B.M. Adipose expression of tumor necrosis factor-alpha: direct role in obesity-linked insulin resistance.Science. 259(5091):87-91, 1993.

HUANG, X.; ZANG, Z. Resistin's, obesity and insulin resistance: the continuing disconnect between rodents and humans. J Endocrinol Invest sv, sn, p. 1-9, 2015.

JANKE, J. et al. Resistin Gene Expression in Human Adipocytes Is Not Related to Insulin Resistance. Obesity Research. v. 10, n. 1, p. 1-5, 2002.

JONES, S.A. et al. The soluble interleukin 6 receptor: mechanisms of production and implications in disease. The FASEB Journal v. 15, sn, p.43-58, 2001.

KERSHAW, E.E.; FLIER, J.S. Adipose Tissue as an Endocrine Organ. The Journal of Clinical Endocrinology \& Metabolism. v. 89, n. 6, p. 2548-2556, 2004.

$\mathrm{KIM}, \mathrm{K}$.E. et al. Blood concentrations of lipopolysaccharide-binding protein, high-sensitivity C-reactive protein, tumor necrosis factor- $a$, and Interleukin- 6 in relation to insulin resistance in young adolescents. Clinica Chimica Acta. 486:115-121, 2018.

LAN, H. et al. Gene Expression Profiles of Nondiabetic and Diabetic Obese Mice Suggest a Role of Hepatic Lipogenic Capacity in Diabetes Susceptibility. DIABETES v. 52, sn, p. 688-700, 2003.

LEE, C.H.; LAM, K.S.L. Obesity-induced insulin resistance and macrophage infiltration of the adipose tissue: a vicious cycle. J Diabetes Investig v.10, n. 1, p. 29-31, 2019.

LEE, Y. et al. Liporegulation in diet-induced obesity. The antisteatotic role of hyperleptinemia. J Biol Chem. 276, $5629-5635,2001$.

LEITE, L.D.; ROCHA, E.D.M.; Brandão-Neto, J. Obesidade: uma doença inflamatória. Revista Ciência \& Saúde. v. 2, n. 2, p. 85-95, 2009. 
LIU, L. et al. Intracerebroventricular leptin regulates hepatic but not peripheral glucose fluxes. J Biol Chem. 273, $31160-31167,1998$.

LUIS, D.A. et al. Visfatina en pacientes obesos, relación con factores de riesgo cardiovascular, un estudio transversal. Elsevier España, S.L. v. 137, n. 5, p.199-203, 2011.

MAGED A.E.W. et al. Serum Apelin and Obesity-Related Complications in Egyptian Children. J. Med. Sci. v. 8, n. 6, p. 1354-1358, 2018.

MARTOS-MORENO, G.A.; Kopchick, J.J.; Argente, J. Adipoquinas en el niño sano y con obesidad. Anales de Pediatría v. 78, n. 3, p. 189.e1- 189.e15, 2013.

MOONISHA, T.M. et al. Evaluation of Leptin as a Marker of Insulin Resistance in Type 2 Diabetes Mellitus. International Journal of Applied and Basic Medical Research. v. 7, n. 3, p. 176-180, 2017.

MORENO-ALIAGA, M.J. et al. Visfatina, apelina y nuevas moléculas del síndrome metabólico. Revista Española de Obesidad. v. 6, n. 4, p. 205-214, 2008.

MORETTO, S.F.; Torres, J.R.P.; Alegre-Maller, A.C.P. Obesidade, resistência à insulina e distúrbios da coagulação: uma revisão da literatura. Revista Thêma et Scientia v. 6, n. 1, p. 101-113, 2016.

MORRISON, C.D. et al. Implications off crosstalk between leptin and insulin signaling during the development of diet-induced obesity. Biochimica et Biophysica Acta. 409-416, 2009.

OWECKI, M. et al. Serum Resistin Concentrations are Higher in Human Obesity but Independent from Insulin Resistance. Exp Clin Endocrinol Diabetes. 119: 117 - 121, 2011.

PETTO, J. et al. Adiponectina: Caracterização, Ação Metabólica e Cardiovascular. International Journal of Cardiovascular Sciences v. 28, n. 3, p.101-109, 2015.

PIÑERO, R. et al. Adiponectin is synthesized and secreted by human and murine cardiomyocytes. FEBS Letters. v. 579, sn, p. 5163-5169, 2005.

PINTO, W.J. A função endócrina do tecido adiposo. Rev. Fac. Ciênc. Méd. Sorocaba. v. 16, n. 3, p. 111 120, 2014.

REHMAN, K.; AKASH, M.S.H. Mechanisms of inflammatory responses and development of insulin resistance: how are they interlinked? Journal of Biomedical Science. 23:87, p. 1-18, 2016.

RIBEIRO FILHO, F.F. et al. Gordura Visceral e Síndrome Metabólica: Mais Que Uma Simples Associação. Arquivos Brasileiros de Endocrinologia \& Metabologia. v. 50, n. 2, p. 230-238, 2006.

ROCHA, V.Z.; FOLCO, E.J. Inflammatory Concepts of Obesity. International Journal of Inflammation. sv, sn, p. 1-14, 2011.

SAVAGE, D.B. et al. Resistin / Fizz3 Expression in Relation to Obesity and Peroxisome ProliferatorActivated Receptor-Action in Humans. Diabetes 2001 in HERMSDORFF, H.H.M.; MONTEIRO, J.B.R. Gordura Visceral, Subcutânea ou Intramuscular: Onde Está o Problema? Arq Bras Endocrinol Metab. v. 48, n. 6, p. 803-811, 2004. 
SCHUSTER, D.P. Obesity and the development of type 2 diabetes: the effects of fatty tissue inflammation. Diabetes, Metabolic Syndrome and Obesity. 3253-262, 2010.

SEUFERT, J. Leptin Effects on Pancreatics $\beta$-Cell Gene Expression and Function. American

TILG, H.; MOSCHEN, A.R. Role of adiponectin and PBEF/visfatin as regulators of inflammation: involvement in obesity-associated diseases. Biochemical Society. v. 14, n. 1, p. 275-288, 2008.

WALDER, K. et al. Leptin inhibits insulin binding in isolated rat adypocites. Jounal of Endocrinology. v. 155, sn, p. 5-7, 1997.

WEYER, C. et al. Hypoadiponectinemia in Obesity and Type 2 Diabetes: Close Association with Insulin Resistance and Hyperinsulinemia. The Journal of Clinical Endocrinology \& Metabolism. v.86, n. 5, p. 1930-1935, 2001.

XU, S.; TSAO, P.S.; YUE, P. et al. Apelin and insulin resistance: another arrow for the quiver? National Institutes of Health. 3(3): 225-231, 2011.

ZAIDI, S.L.; Shirwany, T.A. Relationship Of Serum Resistin With Insulin Resistance And Obesity. J Ayub Med Coll Abbottabad. 27(3):552-5, 2015.

ZULET, M.A. et al. Biomarcadores del estado inflamatorio: nexo de unión con la obesidad y complicaciones asociadas. Nutr Hosp. v. 22, n. 5, p. 511-527, 2007. 